\title{
Fyn kinase regulates dopaminergic neuronal apoptosis in animal and cell models of high glucose $(\mathrm{HG})$ treatment
}

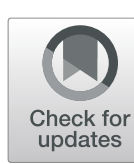

\author{
Changhong Tan ${ }^{1 \dagger}, \mathrm{Xi}_{\mathrm{Li}}{ }^{1 \dagger}$, Xiaoshuai Zhang ${ }^{2}$, Wuxue Peng ${ }^{1}$, Hui Wang ${ }^{1}$, Wen Zhou', Jin Jiang ${ }^{1}$, Lijuan Mo ${ }^{1}$,
} Yangmei Chen ${ }^{1 *}$ and Lifen Chen ${ }^{1 *}$

\begin{abstract}
Background: High glucose $(H G)$ is linked to dopaminergic neuron loss and related Parkinson's disease (PD), but the mechanism is unclear.

Results: Rats and differentiated SH-SY5Y cells were used to investigate the effect of HG on dopaminergic neuronal apoptotic death. We found that a 40-day HG diet elevated cleaved caspase 3 levels and activated Fyn and mTOR/ S6K signaling in the substantia nigra of rats. In vitro, 6 days of HG treatment activated Fyn, enhanced binding between Fyn and mTOR, activated mTOR/S6K signaling, and induced neuronal apoptotic death. The proapoptotic effect of HG was rescued by either the Fyn inhibitor PP1 or the mTOR inhibitor rapamycin. PP1 inhibited mTOR/S6K signaling, but rapamycin was unable to modulate Fyn activation.
\end{abstract}

Conclusions: HG induces dopaminergic neuronal apoptotic death via the Fyn/mTOR/S6K pathway.

Keywords: High glucose, Dopaminergic neuronal apoptosis, Parkinson's disease, Fyn, mTOR

\section{Background}

Diabetes mellitus (DM) is associated with an increased risk for Parkinson's disease (PD) [1, 2]. Dysregulated blood glucose levels have been identified as a risk marker for more rapid disease progression in PD [3]. However, the mechanism whereby this occurs is unclear. Experimental studies have suggested that neuronal apoptosis activation $[4,5]$ may play a crucial role in dopaminergic neuronal loss in the substantia nigra [6]. Interestingly, hyperglycemia in DM patients was reported to induce cortical neuronal apoptosis [7]. In vitro, high glucose (HG) can cause human SH-SY5Y cell apoptosis [8], and this cell line is frequently chosen for PD

\footnotetext{
*Correspondence: cq_neurologist@163.com; lifen_chen@cqmu.edu.cn ${ }^{\dagger}$ Changhong Tan and Xi Liu contributed equally to this work. 'Department of Neurology, The Second Affiliated Hospital of Chongqing Medical University, 74 Linjiang Road, Yuzhong District, Chongqing 400010, China

Full list of author information is available at the end of the article
}

research [9]. Moreover, in a rat model, long-term hyperglycemia induced dopaminergic neurodegeneration and parkinsonian symptomatology [10]. These data indicate that HG may be involved in dopaminergic neuronal apoptosis in PD. However, the mechanism by which HG causes dopaminergic neuronal apoptosis needs further investigation.

Fyn is a nonreceptor tyrosine kinase. Some researchers have reported that Fyn activation promotes apoptosis in cortical and hippocampal neurons [11, 12], and inhibition of Fyn activity inhibits neuronal apoptosis [13]. Moreover, Fyn was reported to induce PD progression in animal models of the disease [14, 15], suggesting a possible role for Fyn in dopaminergic neuronal apoptotic death in PD. Furthermore, Fyn was reported to be involved in glucose metabolism, and Fyn knockout mice exhibit improved glucose tolerance [16]. Therefore, it is plausible that HG-induced dopaminergic neuronal

(c) The Author(s). 2021 Open Access This article is licensed under a Creative Commons Attribution 4.0 International License, which permits use, sharing, adaptation, distribution and reproduction in any medium or format, as long as you give appropriate credit to the original author(s) and the source, provide a link to the Creative Commons licence, and indicate if changes were made. The images or other third party material in this article are included in the article's Creative Commons licence, unless indicated otherwise in a credit line to the material. If material is not included in the article's Creative Commons licence and your intended use is not permitted by statutory regulation or exceeds the permitted use, you will need to obtain permission directly from the copyright holder. To view a copy of this licence, visit http://creativecommons.org/licenses/by/4.0/. The Creative Commons Public Domain Dedication waiver (http://creativecommons.org/publicdomain/zero/1.0/) applies to the data made available in this article, unless otherwise stated in a credit line to the data. 
apoptosis is mediated by Fyn activation, but this has yet to be clarified.

Mammalian target of rapamycin (mTOR)/p70 S6 kinase (S6K) is a well-known regulator of various cellular functions, including apoptosis [17]. mTOR was reported to accelerate neuronal death [18] and to be a risk factor for PD [19]. In a mouse model of PD, levels of phosphorylated mTOR (Ser2448) (p-mTOR) in the substantia nigra were increased [20], and pretreatment with the pmTOR inhibitor rapamycin partially prevented the cell death induced by 6-OHDA [21], exerting a neuroprotective effect in cellular and animal models of PD [22]. Additionally, mTOR/S6K was reported to be activated by HG treatment in differentiated SH-SY5Y cells [23]; however, the mechanism whereby this occurs is unclear. mTOR is also reportedly regulated by Fyn in erythroblasts [24]. Interestingly, Fyn can be activated by HG [25], indicating a possible role for Fyn in HG-activated mTOR. However, whether HG treatment activates mTOR/S6K via Fyn in differentiated SH-SY5Y cells and animal models and leads to neuronal apoptotic death has yet to be clarified. Furthermore, cleaved caspase 3, a marker of cell apoptosis, was reported to be increased by mTOR activation [26].

Thus, we hypothesized that HG treatment activates Fyn, which promotes mTOR phosphorylation by binding and subsequently activating the mTOR/S6K pathway, ultimately inducing dopaminergic neuronal apoptotic death reflected by elevated caspase 3 levels. In this study, Fyn and mTOR/S6K expression and activation levels in a hyperglycemic rat model and HG-treated differentiated SH-SY5Y cells were assessed. Next, we examined whether inhibition of Fyn had a neuroprotective effect against HG-induced dopaminergic neuronal apoptosis via the $\mathrm{mTOR} / \mathrm{S} 6 \mathrm{~K}$ pathway.

\section{Results}

An HG diet elevates cleaved caspase 3 levels and induces activation of Fyn and mTOR/S6K in the substantia nigra of rats

Rats were divided into 2 groups: control and HG diet. After 40 days, fasting glucose levels (HG: $12.40 \pm 6.04$ $\mathrm{mmol} / \mathrm{L}$ vs. control: $4.61 \pm 1.29 \mathrm{mmol} / \mathrm{L}, \quad n=10, p=$ 0.003 ) and glucose levels $2 \mathrm{~h}$ post glucose challenge (HG: $21.89 \pm 9.76 \mathrm{mmol} / \mathrm{L}$ vs. control: $7.50 \pm 1.70 \mathrm{mmol} /$ $\mathrm{L}, \mathrm{n}=10, p=0.001)$ were significantly higher in the HG group than in the control group.

The HG diet also increased cleaved caspase 3 levels in the substantia nigra compared to the controls $(1.739 \pm$ 0.288 vs. $1, n=5, p=0.005$ ) (Fig. $1 \mathrm{~A}$ ). Immunofluorescence staining for cleaved caspase 3 revealed a positive signal for apoptosis in dopaminergic neurons (Fig. 1B). However, TUNEL staining revealed no obviously positive apoptotic dopaminergic neurons in the HG group (Fig. 1C).

Double labeled fluorescence staining suggested that Fyn and mTOR are localized in dopaminergic neurons in the substantia nigra (Fig. 1D). The HG diet increased levels of phosphorylated Fyn on Y-416 (p-Y416) in the substantia nigra of rats compared to controls (1.928 \pm 0.322 vs. $1 n=5, p=0.003)$. However, total protein levels of Fyn were unaffected $(1.008 \pm 0.032$ vs. $1, n=5, p=$ 0.722) (Fig. 1E). Furthermore mRNA levels of Fyn were also unchanged by the HG diet $(0.982 \pm 0.096$ vs. $1, \mathrm{n}=$ $5, p=0.691$ ) (Fig. 1F). Additionally, the HG diet significantly increased the levels of p-mTOR at Ser2448 $(2.259 \pm 0.688$ vs. $1, \mathrm{n}=5, p=0.015)$ and $\mathrm{p}-\mathrm{S} 6 \mathrm{~K}$ at Thr389 (1725 \pm 0.303 vs. $1, n=5, p=0.006)$, while total mTOR and S6K levels were unchanged (Fig. 1G). These data indicated that the HG diet activates the Fyn and mTOR/S6K pathways.

\section{Six-day HG treatment induces apoptotic death and activates Fyn and mTOR/S6K pathways in differentiated SH-SY5Y cells}

Six-day HG treatment significantly increased cleaved caspase 3 levels compared to controls $(3.979 \pm 1.010$ vs. $1, n=5, p=0.003$ ) (Fig. 2A). Cell viability was also significantly decreased in the HG group compared to controls $(2.217 \pm 0.123$ vs. $1.321 \pm 0.068, n=5, p<0.001)$ (Fig. 2B). Annexin V-FITC-positive cells were significantly increased in the HG group compared to controls $(15.31 \pm 3.24 \% \quad$ vs. $2.940 \pm 0.819 \%, \quad n=3, \quad \mathrm{p}=0.003)$ (Figs. 2C).

HG treatment significantly increased levels of p-Y416 compared to controls $(2.426 \pm 0.665$ vs. $1, n=5, p=$ $0.009)$. However, total protein $(1.024 \pm 0.120$ vs. $1, \mathrm{n}=5$, $p=0.668)$ (Fig. 2D) and mRNA levels (1.014 \pm 0.125 vs. $1, \mathrm{n}=3, p=0.862$ ) (Fig. 2E) of Fyn were unchanged in response to $\mathrm{HG}$ treatment. Additionally, 6-day treatment with HG significantly increased the levels of p-mTOR at Ser2448 (2.349 \pm 0.185 vs. $1, \mathrm{n}=5, p<0.001)$ and $\mathrm{p}$-S6K at Thr389 $(2.174 \pm 0.181$ vs. $1, \mathrm{n}=5, \mathrm{p}<0.001)$ in the HG group compared to the control group, while total mTOR and S6K levels remained unchanged (Fig. 2F).

\section{Inhibition of Fyn alleviates HG-induced cell injury by suppressing the mTOR/S6K signaling pathway}

To investigate whether Fyn regulates HG-induced apoptotic cell death by activating mTOR/S6K, the mTOR inhibitor rapamycin and the Fyn inhibitor PP1 were used. Cells were divided into 4 groups: control+DMSO, HG + DMSO, HG + rapamycin and HG + PP1. Elevated cleaved caspase 3 levels induced by HG treatment were rescued by rapamycin $(100 \mathrm{nM})$ administration [22] $(0.833 \pm 0.261$ vs. $2.339 \pm 0.490, n=5, p=0.004)$ or PP1 treatment $(10 \mu \mathrm{M})$ [27] $(0.952 \pm 0.195$ vs. $2.339 \pm 0.490$, 


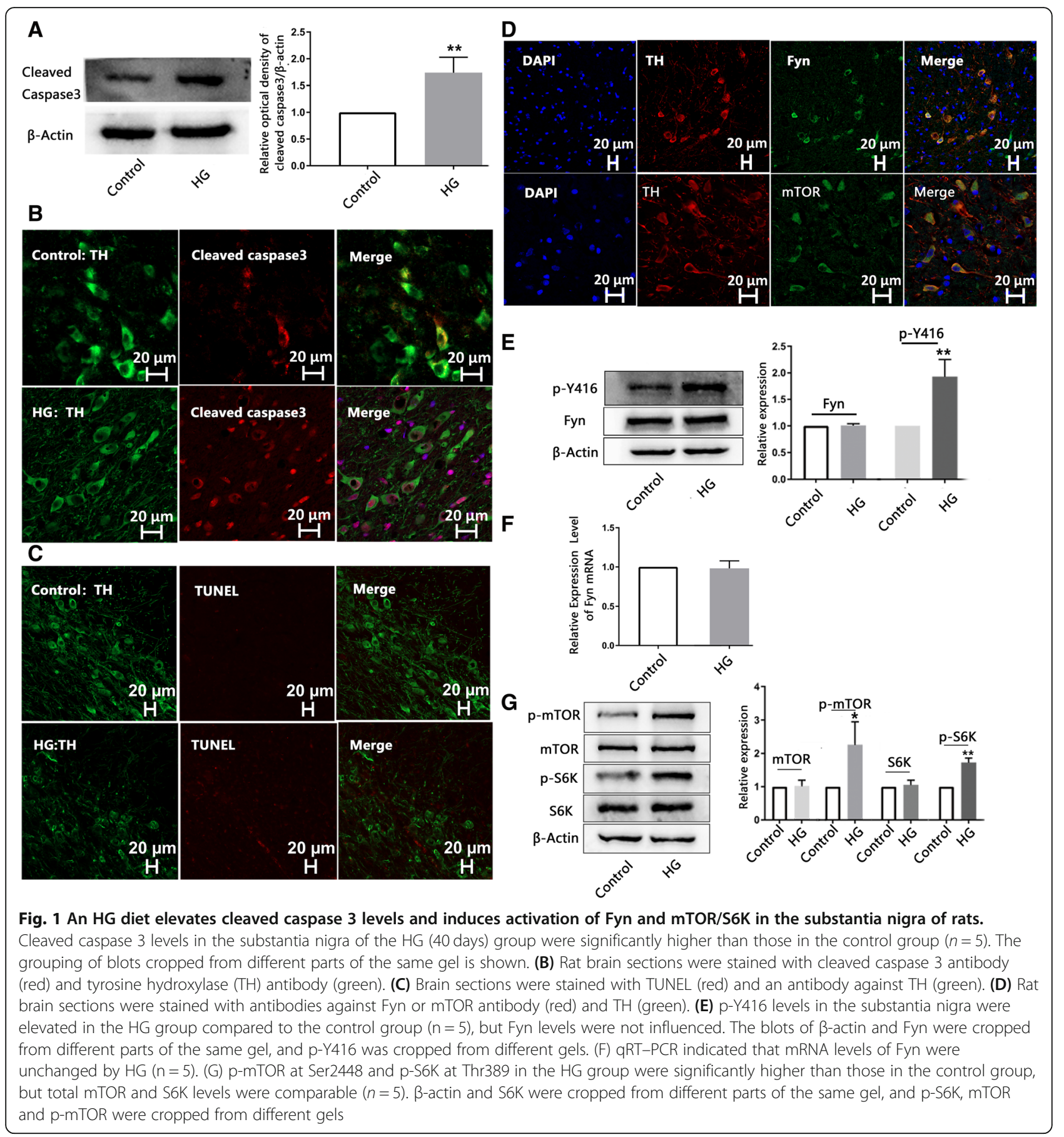

$\mathrm{n}=5, p=0.008)$ (Fig. 3A). PP1 reversed the HG-induced elevation of p-Y416 $(1.325 \pm 0.127$ vs. $2.537 \pm 0.552, n=$ $5, p=0.03$ ) (Fig. 3A) and simultaneously repressed the activation of mTOR $(0.720 \pm 0.196$ vs. $2.113 \pm 0.253, \mathrm{n}=$ $5, p<0.001)$ and S6K $(1.132 \pm 0.551$ vs. $2.746 \pm 0.656$, $\mathrm{n}=5, p=0.017$ ) induced by HG treatment (Fig. 3B). Rapamycin downregulated the levels of p-mTOR $(0.877 \pm 0.090$ vs. $2.113 \pm 0.253, \mathrm{n}=5, p=0.001)$ and $\mathrm{p}$ S6K $(1.420 \pm 0.494$ vs. $2.746 \pm 0.656, \mathrm{n}=5, p=0.039)$ in the $\mathrm{HG}+$ rapamycin group compared to the $\mathrm{HG}+$ DMSO group (Fig. 3B); however, rapamycin treatment did not change $\mathrm{p}-\mathrm{Y} 416$ levels $(2.411 \pm 0.304$ vs. $2.537 \pm$ $0.552, \mathrm{n}=5, p=0.997$ ) (Fig. 3A), suggesting that Fyn acts upstream of the mTOR/S6K signaling pathway.

Furthermore, rapamycin $(8.970 \pm 0.500 \%$ vs. $22.813 \pm$ $1.122 \%, n=3, \mathrm{p}<0.001)$ or PP1 $(7.913 \pm 0.810 \%$ vs. $22.813 \pm 1.122 \%, \mathrm{n}=3, \mathrm{p}<0.001)$ decreased the elevated apoptotic cell death rate induced by HG treatment (Fig. 


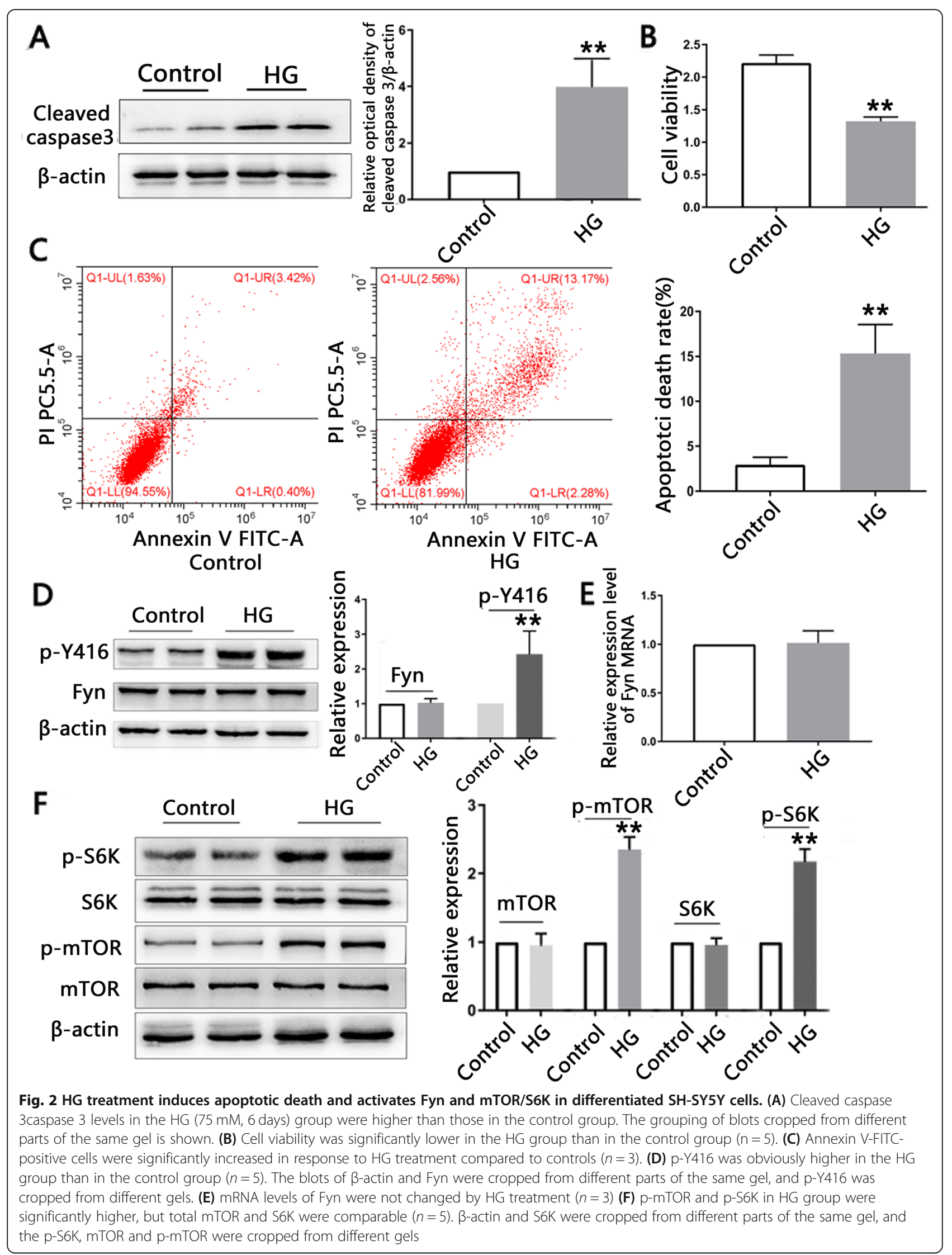



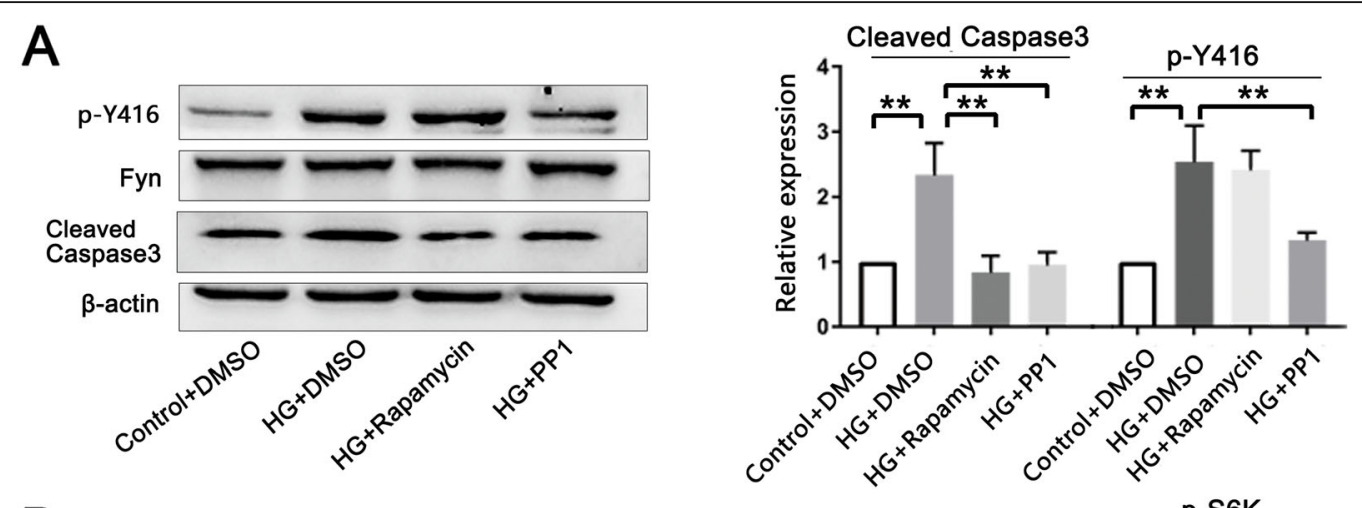

B
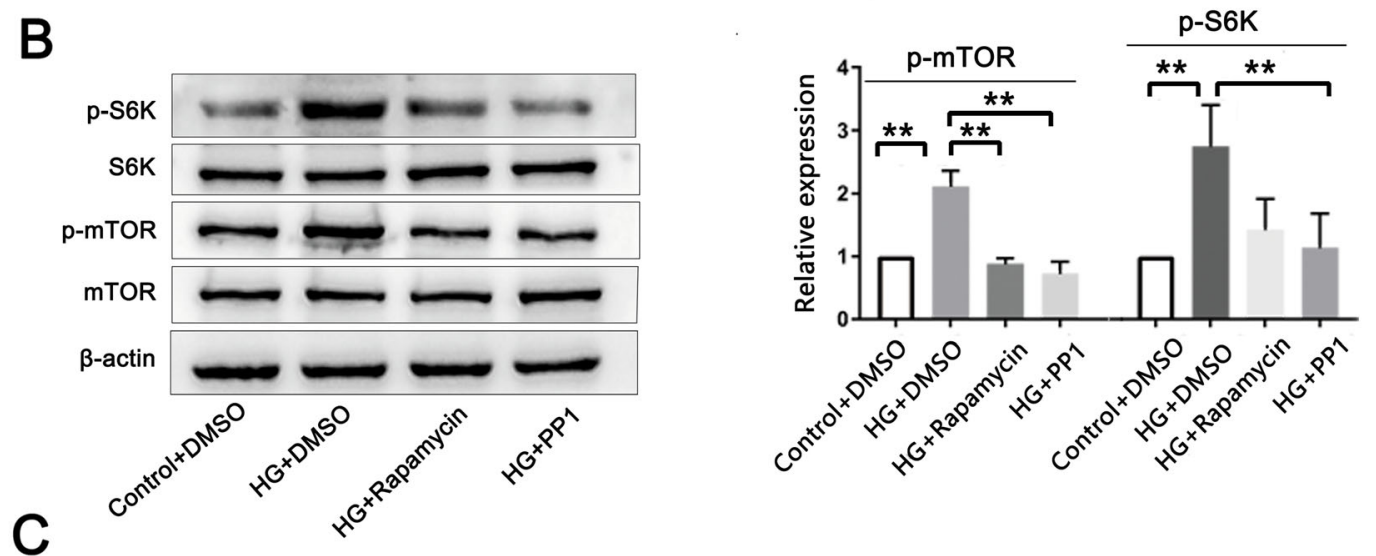

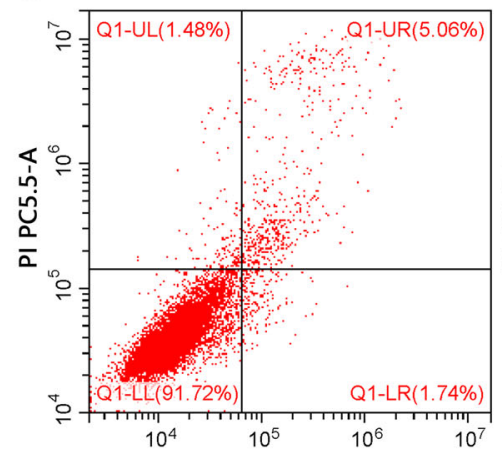

Annexin V FITC-A Control+DMSO

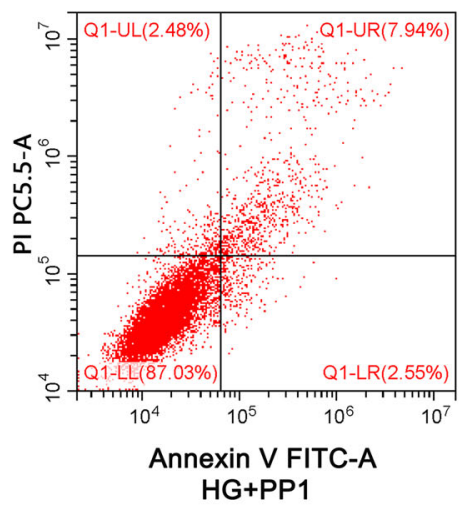

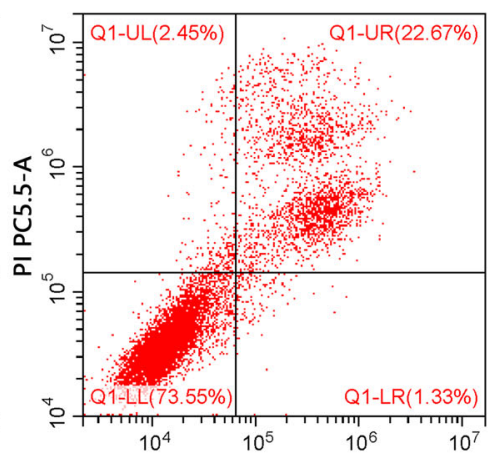

Annexin V FITC-A $\mathrm{HG}+\mathrm{DMSO}$

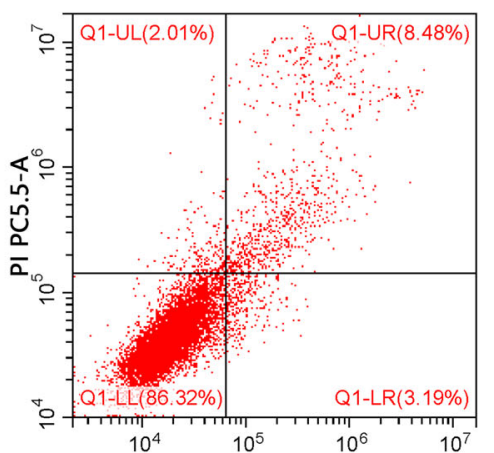

Annexin V FITC-A HG+Rapamycin

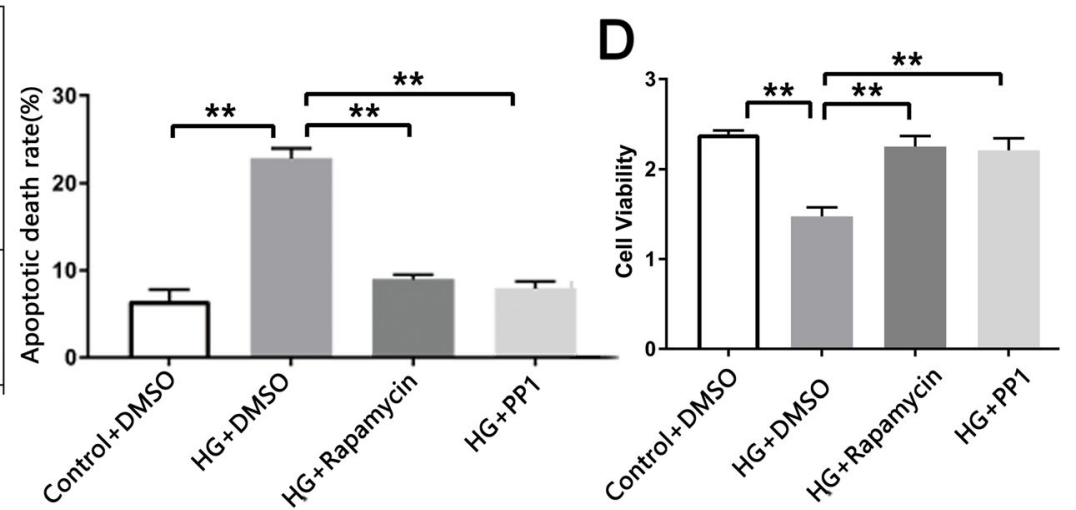

Fig. 3 (See legend on next page.) 
(See figure on previous page.)

Fig. 3 Inhibition of Fyn alleviates HG-induced cell injury by suppressing the mTOR/S6K signaling pathway. (A) Elevated cleaved caspase 3 levels induced by HG were rescued by rapamycin $(100 \mathrm{nM})$ or PP1 $(10 \mu \mathrm{M})$ treatment. PP1 inhibited p-Y416, but rapamycin did not change pY416 levels $(n=5)$. $\beta$-actin, cleaved caspase 3 and Fyn were cropped from different parts of the same gel, and p-Y416 was cropped from a different gel. (B) PP1 or rapamycin inhibited mTOR/S6K $(n=5)$. $\beta$-actin and S6K were cropped from different parts of the same gel, and p-S6K, mTOR and p-mTOR were cropped from different gels. (C) Flow cytometry showed that rapamycin or PP1 decreased the elevated cell apoptotic death induced by HG $(n=3)$. (D) Decreased cell viability in response to HG was rescued by rapamycin or PP1 administration $(n=5)$

3C), and cell viability was rescued by rapamycin or PP1 administration (Fig. 3D), suggesting a protective effect of inhibiting either Fyn or mTOR on HG-induced cell injury.

Fyn may regulate the $\mathrm{mTOR} / \mathrm{S} 6 \mathrm{~K}$ signaling pathway by binding to $\mathrm{mTOR}$

HG-induced apoptosis was mediated by activating the Fyn/mTOR/S6K pathway. However, how Fyn regulates $\mathrm{mTOR} / \mathrm{S} 6 \mathrm{~K}$ signaling is unknown. To address this, we performed quantitative Co-IP. Differentiated SH-SY5Y cells were divided into control and HG groups, and HG treatment elevated mTOR-bound Fyn levels (2.085 \pm 0.342 vs. $1, n=3, p=0.005$ ) (Fig. $4 \mathrm{~A}$ ). The reciprocal experiment using an anti-Fyn antibody also validated that
Fyn bound to mTOR $(2.102 \pm 0.420$ vs. $1, \mathrm{n}=3, p=$ 0.010 ) (Fig. 4B) as mTOR levels were increased in response to HG treatment. These data indicated that HG activates Fyn, enhancing binding between Fyn and mTOR and potentially subsequently activating the Fyn/ mTOR/S6K signaling pathway.

\section{Discussion}

Activated caspase 3 has been observed in the substantia nigra of PD patients and is described as a vulnerability factor in the apoptotic death of dopaminergic neurons $[28,29]$. In our study, cleaved caspase 3 levels were increased in the substantia nigra of rats, and immunofluorescence staining with cleaved caspase 3 revealed a positive signal for apoptosis in dopaminergic neurons.
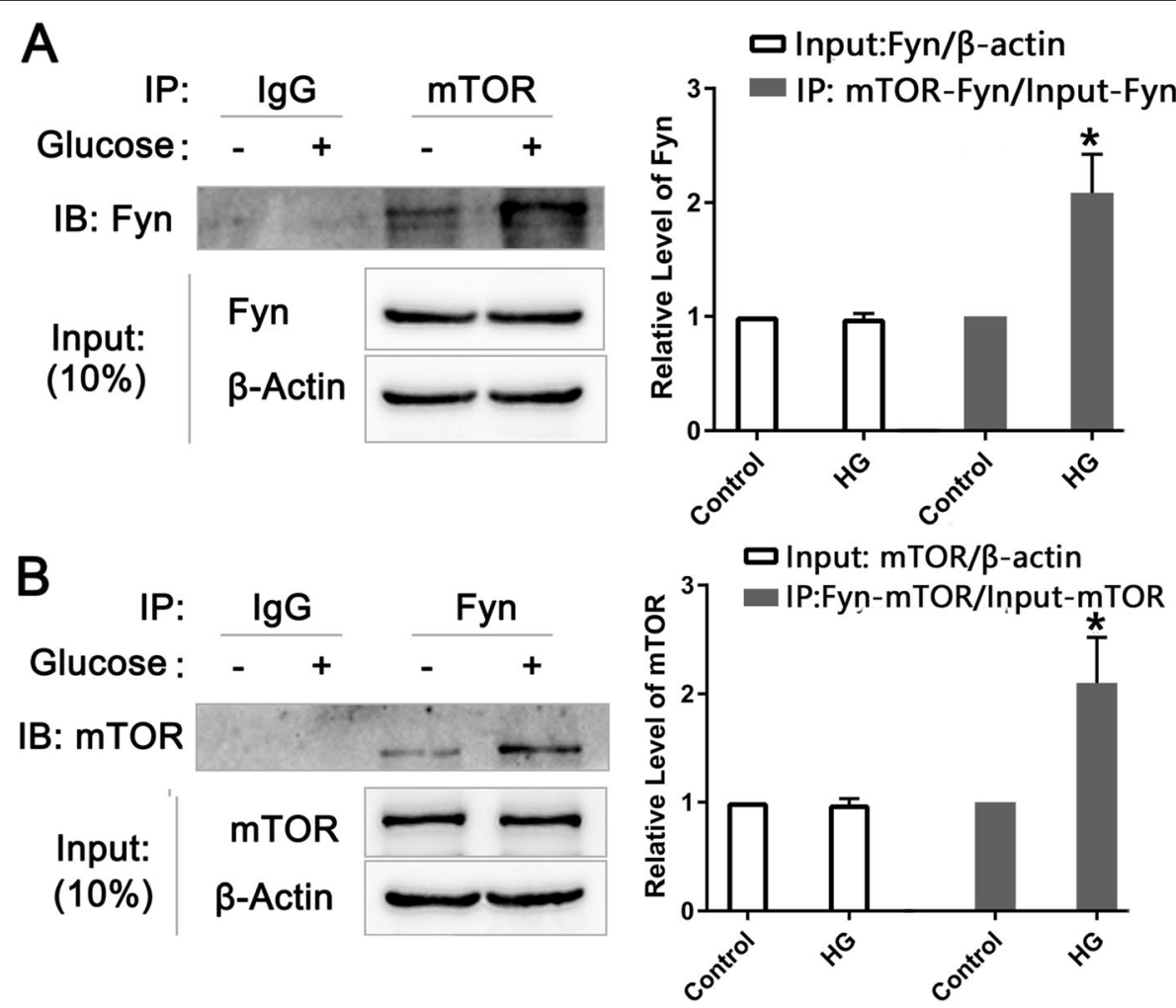

Fig. 4 Fyn may regulate the mTOR/S6K signaling pathway by binding to mTOR. (A) Quantitative Co-IP analysis indicated that HG treatment elevates mTOR-bound Fyn levels, but Fyn/ $\beta$-actin input was not changed $(n=3)$. The grouping of blots of input was cropped from different parts of the same gel, and the IB:Fyn was cropped from a different gel. (B) Reciprocal experiments validated that Fyn-bound mTOR levels were increased by HG treatment, but the mTOR/ $\beta$-actin input was not influenced $(n=3)$. The grouping of blots for the input was cropped from different parts of the same gel, and the IB:mTOR was cropped from a different gel 
However, TUNEL staining, a late-stage apoptotic assay method, did not identify positive apoptotic dopaminergic neurons in the HG diet group. This may be explained by the relatively short duration of hyperglycemia in this study. Previous data has suggested that in vitro, only long-term hyperglycemia leads to neuronal damage [30], and in vivo, 6 but not 3 months of HG treatment caused dopaminergic neuronal loss in rats [10]. Therefore, 40 days of hyperglycemia exposure may not be long enough to cause neuronal death and positive TUNEL staining. Thus, in vitro, a long-term (6 days) HG treatment procedure was used to explore the effect of HG on neuronal cells in this study.

Mechanisms regarding the link between DM and PD incidence are traditionally speculative; these processes share common cellular mechanisms, such as mitochondrial dysfunction, decreased expression of the transcriptional regulator PPAR $\gamma$ coactivator $1 \alpha$, and insulin resistance [31, 32]. However, several studies have suggested that hyperglycemia may be an independent pathophysiological risk factor for PD [33, 34]. A prospective cohort study found that DM patients with high fasting plasma glucose were significantly associated with an increased risk for PD (HR:1.23, 95\%Cl:1.20-1.25) [33], and another study provided evidence that the risk of PD increases due to impaired fasting glucose (IFG) (HR:1.038, 95\%CI:1.009-1.067), a nondiabetic hyperglycemic status, with the authors deducing that the incidence of PD is proportional to the degree or duration of exposure to hyperglycemia rather than simply being dependent on the presence of diabetes [34]. Similarly, we found that a 6-day HG treatment upregulated levels of cleaved caspase 3 and induced apoptotic cell death in differentiated SH-SY5Y cells. These data suggested that the control of hyperglycemia may represent a potential strategy for the management of PD, especially in those with DM. However, in the same study mentioned above [34], patients receiving antidiabetic agents exhibited a high risk for PD. This may be due to an uncontrolled glycemic burden. Moreover, drug use does not imply a substantial intervention in the disease mechanism [34]. Therefore, it is reasonable to speculate that there are other mechanisms involved in the pathogenesis of hyperglycemia leading to PD, and we found that Fyn may represent a new therapeutic target that mediates HG-induced dopaminergic neuronal apoptotic death.

The phosphorylation status of the Y416 residue in the activation loop domain of Fyn was suggested to be a direct indicator of Fyn kinase activation, which can be determined using a p-Y416 antibody [14]. Fyn was reported to be activated by oxidative stress [35], toxic forms of amyloid $\beta$ protein [36] and cellular prion proteins [37]. However, whether Fyn is activated by HG and subsequently play a role in neuronal apoptosis has not been previously studied. In podocytes, high glucose promotes Fyn activation [25]; unfortunately, the authors did not investigate the mechanism by which HG treatment activated Fyn. As a key molecule regulating energy metabolism, Fyn plays a critical role in cellular responses such as insulin signaling [38], in which glucose metabolism is involved. Otherwise, Fyn is reported to be activated by integrins [39], the expression of which can be elevated by HG treatment [26]. Additionally, HG can cause oxidative injury, which can also activate Fyn [35]. However, whether HG activates Fyn via these mechanisms in differentiated SH-SY5Y cells needs further investigation. In this study, we found that HG treatment activated Fyn, characterized by elevated levels of p-Y416 both in vitro and in vivo, while the proapoptotic effect of HG was abolished by the Fyn inhibitor PP1 in vitro, suggesting that Fyn activity plays a crucial role in HGinduced dopaminergic neuronal apoptotic death and may be involved in dopaminergic neuron loss in PD patients with hyperglycemia. However, we detected no change in total Fyn protein or mRNA levels, which may be explained by Fyn exerting its function primarily by relying on kinase activity [39]. These data indicated that the pro-apoptotic effect of HG is mediated by Fyn activation but not Fyn expression, which was in line with findings in another work, with no alterations in total Fyn expression observed in response to HG treatment [25].

Although the molecular pathway that couples Fyn function to dopaminergic neuronal apoptosis is unclear, mTOR is well known as a regulator of various cellular functions, including apoptosis [17]. Levels of p-mTOR (Ser2448) in the substantia nigra are increased [20], and the mTOR inhibitor rapamycin exerts a neuroprotective effect in cell and animal models of PD [22]. Furthermore, knockdown of S6K also potentiated the inhibitory effect of rapamycin on apoptotic cell death in neuronal cells [40]. These studies indicated that mTOR may activate its downstream effector p70S6K, ultimately leading to neuronal apoptosis. The mTOR/S6K signaling pathway was also reported to be activated by HG [23]. We consistently observed that HG treatment activates Fyn/ mTOR/S6K signaling both in vivo and in vitro and that rapamycin suppresses HG-induced neuronal apoptosis in vitro. mTOR is reported to be regulated by several nonreceptor tyrosine kinases, such as Src [41] and spleen tyrosine kinase [42], and it can also be regulated by Fyn [43], which affects glucose metabolism [16]. Thus, we assessed whether activation of mTOR/S6K was induced by Fyn. We found that inhibition of either mTOR or Fyn suppressed HG-induced neuronal apoptosis, and inhibition of Fyn prevented HG-induced activation of mTOR/ S6K signaling; however, mTOR inhibition by rapamycin did not suppress Fyn activation, indicating that Fyn acts upstream of $\mathrm{mTOR} / \mathrm{S} 6 \mathrm{~K}$. These results indicated that 
HG-induced dopaminergic neuronal apoptosis is mediated by activating the Fyn/mTOR/S6K pathway.

Additionally, how Fyn activates the mTOR/S6K signaling pathway is unclear. We found that HG enhances the binding between Fyn and mTOR through quantitative coimmunoprecipitation, and Fyn activation elevates the phosphorylation levels of mTOR. Similarly, Fyn has been reported to promote the phosphorylation of target proteins, such as the semaphorin receptor, NMDAR, NR2A Tau and $\alpha$-synuclein, by binding these factors [39]. Furthermore, phosphorylated Fyn activates mTOR in HEK293T cells [44] and skeletal muscle [43], and knockdown of Fyn inhibits phosphorylation of mTOR in cholangiocarcinoma cell lines [45]. These findings provide evidence that activated Fyn phosphorylates mTOR and subsequently activates the Fyn/mTOR/S6K signaling pathway.

The limitation of this study is that we used PP1 to investigate the functional loss of Fyn; however, there may be additional mechanisms by which PP1 may confer neuroprotective effects. Further experiments knocking down or knocking out Fyn gene expression may support the findings that Fyn-mediated HG induces neuronal apoptotic death more strongly. Although the differentiated SH-SY5Y cells used in this study are frequently chosen for PD research, the SH-SY5Y cell line displays a number of genetic aberrations due to its cancerous origin [9], and primary substantia nigra dopaminergic neurons could be a better choice for developing a PD cell model.

\section{Conclusions}

HG treatment activates Fyn, promotes the interaction between Fyn and mTOR, and subsequently activates Fyn/mTOR/S6K signaling, ultimately inducing dopaminergic neuronal apoptotic death. These results revealed a novel mechanism involved in HG-associated dopaminergic neuronal death. Fyn may represent a therapeutic target for preventing HG-related dopaminergic neuronal loss in PD patients.

\section{Materials and methods}

\section{Animal experiments}

Adult male Sprague-Dawley rats weighing 200 to $300 \mathrm{~g}$ (Experimental Animal Center of Chongqing Medical University) were housed in a specific-pathogen-free facility with a 12-h light/dark cycle and were allowed free access to standard chow and water for 1 week. Then, rats were randomly divided into 2 groups: control $(n=10)$, which remained on a standard diet until the end of the experiment, and HG diet, which was fed regular chow and had continuous free access to water containing 50\% glucose $(n=10)$. Rats were sacrificed by intraperitoneal injection of pentobarbital after a 40-day experimental procedure. Brains were removed for analysis, and their remaining carcasses were transferred to the animal care center.

\section{Glucose tolerance test}

After a 40-day experimental procedure, blood glucose was measured using a glucometer (Roche Diagnostics Scandinavia AB, Bromma, Sweden). Rats were fasted overnight for $16 \mathrm{~h}$. The plasma glucose concentration of tail blood was measured before and $2 \mathrm{~h}$ after intragastric injection of $50 \%$ glucose $(2 \mathrm{~g} / \mathrm{kg}$ body weight).

\section{Antibodies and chemicals}

Anti-tyrosine hydroxylase (TH) (ab112, Abcam, Cambridge, UK); Anti-TH (sc-25,269, Santa Cruz, Santa Cruz, USA); anti-cleaved Caspase 3 (9664; CST, Danvers, USA); Anti-Fyn (ab184276, Abcam, Cambridge, UK); anti-p-Y416 (6943; CST, Danvers, USA); anti-mTOR (2972; CST, Danvers, USA), anti-p-mTOR (Ser2448) (5536; CST, Danvers, USA); anti-p70 S6 Kinase (9202; CST, Danvers, USA); anti-p-S6K(Thr389) (97,596; CST, Danvers, USA); anti- $\beta$-actin (20536-1-AP, Proteintech, Wuhan, China), FITC-conjugated goat anti-rabbit IgG (Proteintech, Wuhan, China); FITC-conjugated donkey anti-mouse (Proteintech, Wuhan, China); Alexa Fluor 555-conjugated donkey anti-rabbit (Beyotime, Haimen, China); PP1 (HY-13084,MCE,New Jersey, USA); rapamycin (HY-10219, MCE, New Jersey, USA); all-trans retinoic acid (RA) (R2625, Sigma-Aldrich, Missouri, USA); glucose $(15,023,021$,Thermofisher, Waltham, USA).

\section{Human SH-SY5Y neuroblastoma cell culture, differentiation and treatment protocol}

SH-SY5Y cells were purchased from Procell Life Science \& Technology Co., Ltd. (Wuhan, China) and cultured in MEM/F12 containing 10\% fetal bovine serum (FBS), 25 $\mathrm{mM}$ glucose and 1\% penicillin-streptomycin (100 units/ $\mathrm{mL}$ ) (Thermo Fisher, Waltham, USA). After plating for $24 \mathrm{~h}$, media containing $10 \mu \mathrm{M}$ RA was used to induce differentiation on day 1 and was continued for 5 days. On day 4, the medium was replaced with MEM/F12 medium containing $1 \% \mathrm{FBS}$ and $10 \mu \mathrm{M}$ RA. On day 6 , differentiated cells were maintained in MEM/F12 medium containing $75 \mathrm{mM}$ glucose or $69.5 \mathrm{mM}$ mannitol plus $5.5 \mathrm{mM}$ glucose for another 6 days. All drugs were dissolved in DMSO. Cells were pretreated with PP1 $(10 \mu \mathrm{M})$ or rapamycin $(100 \mathrm{nM})$ for $1 \mathrm{~h}$ before HG $(75 \mathrm{mM})$ treatment and continued with the following procedures. Media was replaced every 2 days.

\section{Terminal deoxynucleotidyl transferase dUTP nick end labeling (TUNEL) assay}

TUNEL staining of brain sections in the substantia nigra from rats was performed using the One Step TUNEL 
Apoptosis Assay Kit (C1090, Beyotime, Haimen, China). Dopaminergic neurons were labeled with an anti-TH antibody and a related FITC-conjugated secondary antibody. Images were acquired using a laser scanning confocal microscopy (Nikon 1R, Japan).

\section{Cell viability measurement}

MTS analysis was performed using a Celltiter 96 AQueous One Solution Assay Kit (Promega, Madison, USA) to measure cell viability. Briefly, SH-SY5Y cells were seeded into 96-well plates and differentiated with RA for 5 days. After further treatment with drugs (detailed in the cell culture, differentiation and treatment protocol section), cells were incubated with $100 \mu \mathrm{L}$ of newly added media containing $10 \mu \mathrm{L}$ of MTS reagent at $37^{\circ} \mathrm{C}$ for $4 \mathrm{~h}$. The absorption was subsequently measured at $490 \mathrm{~nm}$ using a microplate reader (MultiSkan, GO, Thermo Fisher, Waltham, USA).

\section{Measurement of apoptotic death}

After treatment with drugs, differentiated SH-SY5Y cells were collected and resuspended in phosphate buffer solution. An Annexin V-FITC apoptosis analysis kit (Sungene Biotech, Tianjin, China) was used to identify apoptotic cells. Next, the apoptotic rate was determined using CytoFLEX (Beckman Coulter, California, USA).

\section{Quantitative real-time polymerase chain reaction (qRT- PCR)}

Total RNA from the substantia nigra of brain tissues from rats after a 40-day experimental procedure and drug-treated differentiated SH-SY5Y cells were extracted using RNAiso plus (Takara, Shiga, Japan) and transcribed into complementary DNAs using HiScript 21II Q RT SuperMix for qPCR (+gDNA wiper) (Vazyme, Nanjing, China) on the Applied Biosystems Veriti-Well Thermal Cycler (Thermo Fisher, Waltham, USA). qRTPCR was performed using ChamQ Universal SYBR qPCR MasterMix (Vazyme, Nanjing, China) on the CFX96 Real-Time System (Thermo Fisher, Waltham, USA). Relative gene expression levels were calculated using the $2^{-\Delta \Delta \mathrm{Ct}}$ method [46]. The primer sequences were as follows: Human Fyn: forward: 5'-GGTGTGAA CTCTTCGTCTCATA-3'; reverse: 5'-TGTCCGTGCT TCATAGTCATAA-3'. Rat Fyn: forward: 5'-AGCGAA ACTGACGGAGGAGAGG-3'; reverse: 5'-GTGCTGAG GGGTGGGGTCTG-3'. Actb2 was used as a housekeeping gene for qRT-PCR in this study.

\section{Western blot}

Total proteins were extracted from substantia nigra brain tissues of rats after a 40-day experimental procedure and differentiated SH-SY5Y cells with respective treatments using RIPA (Beyotime, Haimen, China).
Centrifuged protein lysates were mixed with $5 \times$ sodium dodecyl sulfate (SDS) loading buffer and boiled for 10 min. Equal amounts of protein were separated by $8-12 \%$ SDS-PAGE, gels were cut and then transferred to polyvinylidene fluoride (PVDF) membranes (Merck Millipore, Darmstadt, Germany). Membranes were blocked with QuickBlock Blocking Buffer (Beyotime, Haimen, China) for $30 \mathrm{~min}$ at room temperature and then incubated with primary antibodies overnight at $4{ }^{\circ} \mathrm{C}$. $\beta$-actin was used as a housekeeping gene. After washing in Trisbuffered saline with Tween-20 (TBST), membranes were incubated with a horseradish peroxidase-conjugated goat anti-rabbit antibody (A21020 Abkine, Wuhan, China) for $1 \mathrm{~h}$ at room temperature. Proteins on the PVDF membranes were visualized using a chemiluminescent HRP substrate (WBKLS0100, Merck Millipore, Darmstadt, Germany) and scanned using a Fusion-FX7 image analysis system (Vilber Lourmat, Collégien, France).

\section{Coimmunoprecipitation}

Protein extracts from differentiated SH-SY5Y cells treated with HG or not for 6 days were diluted in cell lysis buffer for immunoprecipitation (Beyotime, Haimen, China) and incubated with $2 \mu \mathrm{g}$ of rat IgG (Beyotime, Haimen, China), anti-Fyn antibody, or anti-mTOR antibody overnight at $4{ }^{\circ} \mathrm{C}$. Then, Protein $\mathrm{A} / \mathrm{G}$ agarose (Beyotime, Haimen, China) was added to the mixtures and rotated at $4{ }^{\circ} \mathrm{C}$ for $2 \mathrm{~h}$. The mixtures were centrifuged at $1000 \mathrm{~g}$ for $5 \mathrm{~min}$, and the precipitates were washed with lysis buffer 5 times. Next, the immunoprecipitates were dissolved in $1 \times$ SDS loading buffer and boiled for $10 \mathrm{~min}$. Western blots were performed as described above to verify the interactions between proteins.

\section{Immunofluorescence staining}

Fixed brain tissues were dehydrated in 30\% sucrose and sliced into $10-\mu \mathrm{m}$-thick frozen sections that were immersed in acetone for $15 \mathrm{~min}$ at $4{ }^{\circ} \mathrm{C}$ and permeabilized in $0.4 \%$ Triton $\mathrm{X}-100$. Then, the sections were blocked with normal donkey serum at room temperature for $1 \mathrm{~h}$ after antigen retrieval. Sections were incubated with $\mathrm{TH}$ and Fyn, mTOR or cleaved caspase 3 antibody at $4{ }^{\circ} \mathrm{C}$ overnight. The sections were then washed with PBS again and incubated with the corresponding secondary fluorescent antibody in darkness at room temperature for $1 \mathrm{~h}$. Then, the sections were stained with DAPI at $37^{\circ} \mathrm{C}$ for $10 \mathrm{~min}$ and mounted with antifade mounting media. Images were acquired using laser scanning confocal microscopy (Nikon 1R, Japan).

\section{Statistical analysis}

All data were assessed for distribution, and if the data were normally distributed, they are presented as the 
means \pm standard deviation (SD). At least three replicated independent experiments were performed. Data analysis and graph creation were performed using SPSS 20.0 software (IBM, Armonk, USA) and GraphPad Prism 6.01 (GraphPad software, La Jolla, USA). The differences between 2 groups were analyzed using Student's t-test. For comparisons of 3 or more groups, one-way ANOVA with Bonferroni's or Dunnett's T3 post hoc analysis was used. $p<0.05$ was considered statistically significant.

\section{Acknowledgements}

Not applicable.

\section{Authors' contributions}

Conceptualization, L.C., Y.C., C.T., and X.L. Methodology, X.Z., C.T., X.L., and W.P. Formal analysis, L.C., C.T., and X.L. Investigation, C.T., X.L., X.Z., W.P., H.W., W.Z., J.J., and L.M. Writing-original draft, C.T., and X.L. Writing-review \& editing, L.C., and Y.C. Visualization, C.T., and X.L. Supervision, L.C., and Y.C. Project administration, L.C., Y.C., X.L. Funding acquisition, L.C. All authors read and approved the final manuscript.

\section{Funding}

This study was funded by the National Natural Science Foundation of China (81771391 and 82001367), and was supported by Kuanren Talents Program of the Second Affiliated Hospital of Chongqing Medical University.

\section{Availability of data and materials}

All data generated or analysed during this study are included in this published article.

\section{Declarations}

\section{Ethics approval and consent to participate}

All procedures performed in studies were approved by Ethics Committee of Chongqing Medical University, and was reported in accordance with ARRIVE guidelines. All methods are performed in accordance with the Regulations of Chongqing Medical University on the administration of laboratory animals (2005)

\section{Consent for publication}

Not applicable.

\section{Competing interests}

The authors declare that they have no competing interests.

\section{Author details}

'Department of Neurology, The Second Affiliated Hospital of Chongqing Medical University, 74 Linjiang Road, Yuzhong District, Chongaing 400010, China. ${ }^{2}$ Chongqing Medical University, Chongqing 400010, China.

Received: 28 May 2021 Accepted: 23 November 2021

Published online: 04 December 2021

\section{References}

1 Xu Q, Park Y, Huang X, Hollenbeck A, Blair A, Schatzkin A, et al. Diabetes and risk of Parkinson's disease. Diabetes Care. 2011;34(4):910-5. https://doi. org/10.2337/dc10-1922.

2 Sun Y, Chang YH, Chen HF, Su YH, Su HF, Li CY. Risk of Parkinson disease onset in patients with diabetes: a 9-year population-based cohort study with age and sex stratifications. Diabetes Care. 2012;35(5):1047-9. https:// doi.org/10.2337/dc11-1511.

3 Mollenhauer B, Zimmermann J, Sixel-Döring F, Focke NK, Wicke T, Ebentheuer J, et al. Baseline predictors for progression 4 years after Parkinson's disease diagnosis in the De novo Parkinson cohort (DeNoPa). Mov Disord. 2019;34(1):67-77. https://doi.org/10.1002/mds.27492.

4 Simunovic F, Yi M, Wang Y, Macey L, Brown LT, Krichevsky AM, et al. Gene expression profiling of substantia nigra dopamine neurons: further insights into Parkinson's disease pathology. Brain. 2009;132(7):1795-809. https://doi org/10.1093/brain/awn323.
5 Michel PP, Hirsch EC, Hunot S. Understanding dopaminergic cell death pathways in Parkinson disease. Neuron. 2016;90(4):675-91. https://doi.org/1 0.1016/j.neuron.2016.03.038.

6 Kalia LV, Lang AE. Parkinson's disease. Lancet. 2015;386(9996):896-912. https://doi.org/10.1016/S0140-6736(14)61393-3.

7 Den Heijer T, Vermeer SE, Van Dijk EJ, et al. Type 2 diabetes and atrophy of medial temporal lobe structures on brain MRI. Diabetologia. 2003;46(12): 1604-10. https://doi.org/10.1007/s00125-003-1235-0.

8 Onphachanh X, Lee HJ, Lim JR, et al. Enhancement of high glucose-induced PINK1 expression by melatonin stimulates neuronal cell survival: Involvement of MT (2) /Akt/NF-kB pathway. J Pineal Res. 2017;63. https://doi org/10.1111/jpi.12427.

9 Xicoy H, Wieringa B, Martens GJ. The SH-SY5Y cell line in Parkinson's disease research: a systematic review. Mol Neurodegener. 2017;24(12):10. https://doi. org/10.1186/s13024-017-0149-0.

10 Renaud J, Bassareo V, Beaulieu J, Pinna A, Schlich M, Lavoie C, et al. Dopaminergic neurodegeneration in a rat model of long-term hyperglycemia: preferential degeneration of the nigrostriatal motor pathway. Neurobiol Aging. 2018;69:117-28. https://doi.org/10.1016/j. neurobiolaging.2018.05.010.

11 Yu W, Gowda M, Sharad Y, Singh SA, Sesti F. Oxidation of KCNB1 potassium channels triggers apoptotic integrin signaling in the brain. Cell Death Dis. 2017;8(4):e2737. https://doi.org/10.1038/cddis.2017.160.

12 Lambert MP, Barlow AK, Chromy BA, et al. Diffusible, nonfibrillar ligands derived from Ab1-42 are potent central nervous system neurotoxins. Proc Natl Acad Sci U S A. 1998;95:6448-53. https://doi.org/10.1073/pnas.95.11.6448.

13 Liang $X$, Yao Y, Lin Y, Kong L, Xiao H, Shi Y, et al. Panaxadiol inhibits synaptic dysfunction in Alzheimer's disease and targets the Fyn protein in APP/PS1 mice and APP-SH-SY5Y cells. Life Sci. 2019;221:35-46. https://doi. org/10.1016/.j.lfs.2019.02.012.

14 Panicker N, Saminathan H, Jin H, Neal M, Harischandra DS, Gordon R, et al. Fyn kinase regulates microglial Neuroinflammatory responses in cell culture and animal models of Parkinson's disease. J Neurosci. 2015;35(27):10058-77. https://doi.org/10.1523/JNEUROSCI.0302-15.2015.

15 Panicker N, Sarkar S, Harischandra DS, Neal M, Kam TI, Jin H, et al. Fyn kinase regulates misfolded a-synuclein uptake and NLRP3 inflammasome activation in microglia. J Exp Med. 2019;216(6):1411-30. https://doi.org/10.1 084/jem.20182191.

16 Bastie CC, Zong H, Xu J, Busa B, Judex S, Kurland IJ, et al. Integrative metabolic regulation of peripheral tissue fatty acid oxidation by the SRC kinase family member Fyn. Cell Metab. 2007;5(5):371-81. https://doi.org/10.1 016/..cmet.2007.04.005.

17 Pene F, Claessens YE, Muller O, Viguié F, Mayeux P, Dreyfus F, et al. Role of the phosphatidylinositol 3-kinase/Akt and mTOR/P70S6-kinase pathways in the proliferation and apoptosis in multiple myeloma. Oncogene. 2002; 21(43):6587-97. https://doi.org/10.1038/sj.onc.1205923.

18 Sarkar S, Ravikumar B, Floto RA, Rubinsztein DC. Rapamycin and mTORindependent autophagy inducers ameliorate toxicity of polyglutamineexpanded huntingtin and related proteinopathies. Cell Death Differ. 2009; 16(1):46-56. https://doi.org/10.1038/cdd.2008.110.

19 Ahmed I, Liang Y, Schools S, Dawson VL, Dawson TM, Savitt JM. Development and characterization of a new Parkinson's disease model resulting from impaired autophagy. J Neurosci. 2012;32(46):16503-9. https:// doi.org/10.1523/JNEUROSCI.0209-12.2012.

20 Nordströma U, Beauvais G, Ghosh A, et al. Progressive nigrostriatal terminal dysfunction and degeneration in the engrailed 1 heterozygous mouse model of Parkinson's disease. Neurobiol Dis. 2015;73:70-82. https://doi.org/1 0.1016/j.nbd.2014.09.012.

21 Alquézar C, Barrio E, Esteras N, de la Encarnación A, Bartolomé F, Molina JA, et al. Targeting cyclin D3/CDK6 activity for treatment of Parkinson's disease. J Neurochem. 2015;133(6):886-97. https://doi.org/10.1111/jnc.13070. Epub 2015 Mar 15.

22 Malagelada C, Jin ZH, Jackson-Lewis V, Przedborski S, Greene LA. Rapamycin protects against neuron death in in vitro and in vivo models of Parkinson's disease. J Neurosci. 2010;30(3):1166-75. https://doi.org/10.1523/JNEUROSCl.3 944-09.2010.

23 Nie SD, Li X, Tang CE, Min FY, Shi XJ, Wu LY, et al. High glucose forces a positive feedback loop connecting ErbB4 expression and mTOR/S6K pathway to aggravate the formation of tau hyperphosphorylation in differentiated SH-SY5Y cells. Neurobiol Aging. 2018;67:171-80. https://doi. org/10.1016/j.neurobiolaging.2018.03.023. 
24 Beneduce E, Matte A, De Falco L, et al. Fyn kinase is a novel modulator of erythropoietin signaling and stress erythropoiesis. Am J Hematol. 2019;94(1) 10-20. https://doi.org/10.1002/ajh.25295.

25 Lv Z, Hu M, Ren X, Fan M, Zhen J, Chen L, et al. Fyn mediates high glucoseinduced actin cytoskeleton reorganization of podocytes via promoting ROCK activation in vitro. J Diabetes Res. 2016;2016:5671803-13. https://doi. org/10.1155/2016/5671803.

26 Wang $Y$, Zhao M, Shang L, Zhang Y, Huang C, He Z, et al. Homer1a protects against neuronal injury via PI3K/AKT/mTOR signaling pathway. Int J Neurosci. 2020;130(6):621-30. https://doi.org/10.1080/00207454.2019.170253 5.

27 Watcharasit $\mathrm{P}$, Tucholski J, Jope RS. Src family kinase involvement in muscarinic receptor-induced tyrosine phosphorylation in differentiated $\mathrm{SH}$ SY5Y cells. Neurochem Res. 2001;26(7):809-16. https://doi.org/10.1023/a:1 011612118779

28 Hartmann A, Hunot S, Michel PP, Muriel MP, Vyas S, Faucheux BA, et al. Caspase-3: a vulnerability factor and final effector in apoptotic death of dopaminergic neurons in Parkinson's disease. Proc Natl Acad Sci U S A. 2000:97(6):2875-80. https://doi.org/10.1073/pnas.040556597.

29 Tatton NA. Increased caspase 3 and Bax immunoreactivity accompany nuclear GAPDH translocation and neuronal apoptosis in Parkinson's disease. Exp Neurol. 2000;166(1):29-43. https://doi.org/10.1006/exnr.2000.7489.

30 Tomlinson DR, Gardiner NJ. Glucose neurotoxicity. Nat Rev Neurosci. 2008; 9(1):36-45. https://doi.org/10.1038/nrn2294.

31 Aviles-Olmos I, Limousin P, Lees A, Foltynie T. Parkinson's disease, insulin resistance and novel agents of neuroprotection. Brain. 2013;136(2):374-84. https://doi.org/10.1093/brain/aws009.

32 Santiago JA, Potashkin JA. Shared dysregulated pathways lead to Parkinson's disease and diabetes. nTrends Mol Med. 2013;19:176-86. https:// doi.org/10.1016/j.molmed.2013.01.002.

33 Nam GE, Kim SM, Han K, Kim NH, Chung HS, Kim JW, et al. Metabolic syndrome and risk of Parkinson disease: a nationwide cohort study. PLoS Med. 2018;15(8):e1002640. https://doi.org/10.1371/journal.pmed.1002640.

34 Rhee SY, Han KD, Kwon H, Park SE, Park YG, Kim YH, et al. Association between glycemic status and the risk of Parkinson disease: a Nationwide population-based study. Diabetes Care. 2020;43(9):2169-75. https://doi.org/1 0.2337/dc19-0760

35 Saminathan $\mathrm{H}$, Asaithambi A, Anantharam V, Kanthasamy AG, Kanthasamy A. Environmental neurotoxic pesticide dieldrin activates a non receptor tyrosine kinase to promote $\mathrm{PKC} \delta$-mediated dopaminergic apoptosis in a dopaminergic neuronal cell model. Neurotoxicology. 2011;32(5):567-77. https://doi.org/10.1016/j.neuro.2011.06.009.

36 Um JW, Nygaard HB, Heiss JK, Kostylev MA, Stagi M, Vortmeyer A, et al. Alzheimer amyloid- $\beta$ oligomer bound to postsynaptic prion protein activates Fyn to impair neurons. Nat Neurosci. 2012;15(9):1227-35. https:// doi.org/10.1038/nn.3178

37 Li C, Götz J. Somatodendritic accumulation of tau in Alzheimer's disease is promoted by Fyn-mediated local protein translation. EMBO J. 2017;36(21): 3120-38. https://doi.org/10.15252/embj.201797724.

38 Lee CG, Koo JH, Kim SG. Phytochemical regulation of Fyn and AMPK signaling circuitry. Arch Pharm Res. 2015;38(12):2093-105. https://doi.org/1 0.1007/s12272-015-0611-X.

39 Matrone C, Petrillo F, Nasso R, Ferretti G. Fyn tyrosine kinase as harmonizing factor in neuronal functions and dysfunctions. Int J Mol Sci. 2020;21(12): 4444. https://doi.org/10.3390/ijms21124444.

40 Xu C, Liu C, Liu L, Zhang R, Zhang H, Chen S, et al. Rapamycin prevents cadmium-induced neuronal cell death via targeting both mTORC1 and mTORC2 pathways. Neuropharmacology. 2015;97:35-45. https://doi.org/10.1 016/j.neuropharm.2015.05.008.

41 Pal R, Palmieri M, Chaudhury A, Klisch TJ, di Ronza A, Neilson JR, et al. Src regulates amino acid-mediated mTORC1 activation by disrupting GATOR1rag GTPase interaction. Nat Commun. 2018;9(1):4351. https://doi.org/10.103 8/s41467-018-06844-4.

42 Gao P, Qiao X, Sun H, Huang Y, Lin J, Li L, et al. Activated spleen tyrosine kinase promotes malignant progression of oral squamous cell carcinoma via mTOR/S6 signaling pathway in an ERK1/2-independent manner. Oncotarget. 2017;8(48):83900-12. https://doi.org/10.18632/oncotarget.19911.

43 Yamada E, Bastie CC, Koga H, Wang Y, Cuervo AM, Pessin JE. Mouse skeletal muscle fiber-type-specific macroautophagy and muscle wasting are regulated by a Fyn/STAT3/Nps34 signaling pathway. Cell Rep. 2012;1(5):55769. https://doi.org/10.1016/j.celrep.2012.03.014
44 Wang $Y$, Yamada E, Zong H, Pessin JE. Fyn activation of mTORC1 stimulates the IRE1a-JNK pathway, leading to cell death. J Biol Chem. 2015;290(41): 24772-83. https://doi.org/10.1074/jbc.M115.687020.

45 Lyu SC, Han DD, Li XL, et al. Fyn knockdown inhibits migration and invasion in cholangiocarcinoma through the activated AMPK/mTOR signaling pathway. Oncol Lett. 2018;15(2):2085-90. https://doi.org/10.3892/ol.2017. 7542.

46 Schmittgen TD, Livak KJ. Analyzing real-time PCR data by the comparative C(T) method. Nat Protoc. 2008;3(6):1101-8. https://doi.org/10.1038/nprot.2 008.73

\section{Publisher's Note}

Springer Nature remains neutral with regard to jurisdictional claims in published maps and institutional affiliations.
Ready to submit your research? Choose BMC and benefit from:

- fast, convenient online submission

- thorough peer review by experienced researchers in your field

- rapid publication on acceptance

- support for research data, including large and complex data types

- gold Open Access which fosters wider collaboration and increased citations

- maximum visibility for your research: over $100 \mathrm{M}$ website views per year

At $\mathrm{BMC}$, research is always in progress.

Learn more biomedcentral.com/submissions 